\title{
SAM-e AND ITS THERAPEUTIC PRINCIPLES
}

\author{
Shiva Shankar Varganti \\ Dept Of Pharmaceutical Technology, Anurag Pharmacy Collage, Kodad, Nalgonda District, A.P., India-508206 \\ Author's Mail id shivashankar.varganti@gmail.com
}

Received 09 Dec 2012; Review Completed 09 Jan 2013; Accepted 12 Jan 2013, Available online 15 Jan 2013

\begin{abstract}
S-Adenosylmethionine(SAM-e) which is an well-knownsuper nutrient in countries like Europe , Italy, but unfortunately it is unknown in many countries, which is mainlyavailable as nutrient supplement, SAM-e though it is not a completely new compound but can be found in our tissues performing trans methylation reaction(methyl donor) with Iupac name as [(3S)-3amino-3 carboxypropyl] (\{[(2S,3S,4R,5R)-5-(6-amino-9H-purin-9-yl)-3,4-dihydroxyoxolan-2-yl ]methyl $\})$ methylsulfanium .SAM-e stands first among all other drugs in treating depression, cirrhosis, osteoarthritis, Alzheimer disease, Though it was discovered in 1952 it self but it does not came in to lime light, much more studies are to be conducted to evaluate it uses and even the way it works can be inspiring to us in preparing its derivatives. Alcoholiccirrhosis patients when given SAM-e miraculously cured just by increasing the regeneration efficacy of the liver, this article mainly discuss the uses of SAMe and the principle mechanism involved in it.
\end{abstract}

Synonyms and Trade Names: S-Adenosylmethionine; active methionine; ademetionine; adenosyl-L-methionine; methioninyladenylate; AdoMet; Donamet; SAMe, Sam-e; S. AmetDisulfateditosylate salt: Gumbaral, Samyr

\section{INTRODUCTION}

SAM-e is a nutrient supplement used for the relief of depression. Osteoarthritis, liver cirrhosis mainly reverses the alcoholic side effects.it was first described in 1952 and has been available in the United States as an over the counter supplementsince 1999; in Europe, it is a prescription medicine ${ }^{1}$ since 1975 , where it is used to treat arthritis and depression. ${ }^{2}$

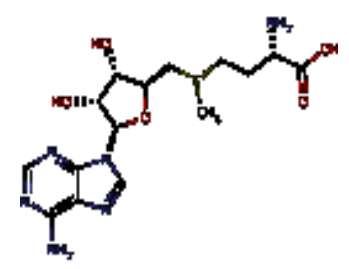

it was first developed as a pharmaceutical by an Italian firm in the early 1970s. To date, it remains one of the most widely prescribed antidepressants in Italy. ${ }^{3}$ it must be remembered that SAMe is not considered a drug in the United States and is therefore not subject to federalregulations. (In contrast, Samyr is a prescription drug in Italy and is available in $200 \mathrm{mg}$ and $400 \mathrm{mg}$ doses.)the iupac name is[(3S)-3-amino-3carboxypropyl](\{[(2S,3S,4R,5R)-5-(6-amino-9H-purin-9yl)-3,4-dihydroxyoxolan-2-yl]methyl $\}$ )methylsulfanium . Recent testing by ConsumerLab.com of over-the-counter brands of SAMe in the United States found, on average, that for 6 of the 13 brands tested, less than half the amount of SAMe stated on the label was actually present.

\section{ROLE OF SAM-e IN OUR BODY}

SAM-e is found in almost every tissue in the body in humans and other mammals, formed by an enzyme (methionine S-adenosyltransferase (MAT)) catalyzed reaction between methionine and ATP (Osman et al., 1993; LEF Magazine, 1997) and Methionine adenosyltransferase (MAT) which is an essential cellular enzyme that catalyzes the formation ofSadenosylmethionine (SAM-e), the principal biological methyl donor and the ultimate source of the propyl aminemoiety used in polyamine biosynthesis. ${ }^{4}$ It also plays a role in cellular metabolism as a methyl donor for transmethylation reactions and also acts as the amino propyl donor in the biosynthesis of polyamines ${ }^{[5,6]}$.

\section{GENERAL MECHANISM OF ACTION}

SAM-e is a ubiquitous methyl-donor molecule located throughout the body. It plays a keyrole in numerous metabolic pathways that involve the transfer of methyl groups. SAMeis not sufficiently available in our diet, but it is formed in the body by thecombination of adenosine triphosphate (ATP) and the amino-acid methionineSAM-e then donates its methyl group to any of a wide range of molecules andis subsequently transformed to homocysteine $^{.7}$

it is mainly involved in SAM CYCLE (The reactions that produce, consume, and regenerate SAM are called the SAM cycle). In the first step of this cycle, the SAMdependent methylase that use SAM as a substrate produce s-adenosylhomocysteine as a product. This is hydrolysed to homocysteine and adenosine by Sadenosylhomocysteine hydrolase and the homocysteine recycled back to methionine through transfer of a methyl group from 5-methyltetrahydrofolate, by one of the two classes of methionine synthases This methionine can then be converted back to SAM, completing the cycle.

\section{PHARMACOLOGY}

Oral SAMe has a very low bioavailability, estimated to be $<1 \%$, so its usefulness as anoral agent is open to question. ${ }^{8}$ few recent studies reveals that $71 \%$ of the patients treatedwith oral SAM-e had a rise in their serum SAM-e concentrations ${ }^{9}$ Parenterallyadministered SAM-e does appear to cross the blood brain barrier. ${ }^{10}$ The half- 
life,metabolism, and excretion of SAM-e have not been well defined and much more studies are to be done to confirm it.

\section{Role of SAMe in treating depression}

SAMe has been shown to decrease depression, ${ }^{11 .}$ It has been hypothesized that the antidepressant effects of SAMe may result from its role as a methyl donor to biogenic amines which influence neurotransmitter metabolism, and from its role in the methylation of membrane phospholipids which modify membrane fluidity and receptor function (Bottiglieri\& Hyland, 1994; Cestaro, 1994; Cowley \& Underwood, 1999).

The antidepressant effects of SAMe were first suggested by Pinzello and Andreoli (1972). Since then, researchers have published some 40 open and double-blinded studies evaluating the efficacy of SAMe supplements for the treatment of depressive disorders in roughly 1,400 subjects. Several studies have shown that SAMe can produce clinical improvement in depressed subjects as effectively as classic tricyclic antidepressants. SAMe also demonstrated antidepressant activity in several predictive models

in mice and rats (Baldessarini, 1987; De Leo, 1987; Kaganet al., 1990; Rosenbaum et al., 1990; Czyraket al., 1992; Bressa, 1994; Benelliet al., 1999; Cowley and Underwood, 1999).

SAMe has not been more effective than prescription antidepressants, but it is clearly less toxic than the tricyclics and MAO inhibitors. Until large clinical trials confirm the results seen from the limited European studies, however, it is unlikely that American doctors will recommend SAM-e to severely depressed persons (Cowley \& Underwood, 1999).

\section{Role in reversing liver injury caused due to alcohol.}

Many studies indicated that SAM-e has reversed the alcoholic injury, and it can be successfully to boost liver.Hepatic SAM levels are decreased in animal models of alcohol liver injury and in patients with alcohol liver disease or viral cirrhosis by acting against alcohol and cytochrome P450 2E1- dependent cytotoxicity both in vitro and in vivo. SAM-e, at high concentrations, inhibits CYP2E1 catalytic activity, lowering formation of $\operatorname{ROS}^{12}$

Animal studies and clinical trials in humans have shown that SAMe, administered orally or by injection, alleviates signs and/or symptoms of liver disease caused by alcohol (humans, rats, and baboons) (Micaliet al., 1983; Feoet al., 1986; Lieberet al., 1990); toxic chemicals, including carbon tetrachloride (rats) (Varela-Moreiraset al., 1995) and hexachlorobenzene (rats) (Cantoniet al., 1990); nonsteroidal anti-inflammatory drugs (NSAIDs), including acetaminophen (mice) (Bray et al., 1992); and cyclosporin A (rats) (Galánet al., 1999). SAMe also alleviated estrogen-induced liver problems (e.g., cholestasis associated with pregnancy) (Almasioet al., 1990; Frezza\&Terpin, 1992; Osman et al., 1993; Floreaniet al., 1996) and hepatic necrosis in rats from methyl deficient diets (Chawlaet al., 1998).

Role in Alzemer disease.
Alzheimer disease, is the most common form of dementia. There is no cure for the disease, which worsens as it progresses, and eventually leads to death the cause and progression of Alzheimer's disease are not well understood. Research indicates that the disease is associated with plaques and tangles in the brain. Current treatments only help with the symptoms of the disease. But SAM-e shows its role effectively in reducing the cause of it as the antibody accumulation is one of the reason foAlzheimer's to occur so it works by reducing the antibody accumulation

\section{Mechanism of action}

balance of presenilin activity or of their expression could be primarily responsible for $\mathrm{Ab}$ accumulation. The progressive SAM reduction observed in the elderly and the consequent methylation decrease, possibility of therapeutically reducing $\mathrm{Ab}$ production. It is unclear whether $\mathrm{Ab}$ accumulation is due to its overproduction or to a clearance defect. However, the reduction of $\mathrm{Ab}$ formation has a good chance of preventing AD. ${ }^{13}$

\section{Osteoarthritis}

SAM-e has a comparable effect to that of NSAIDs in reducing pain and functional limitation. ${ }^{14}$ Researchers discovered the potential usefulness of SAM-e for treating osteoarthritis by accident. They were studying SAM-e's effect on depression when the patients they were following reported an unexpected improvement in their osteoarthritis symptom. SAMe is critical for manufacturing joint cartilage and for maintaining neural cell membrane function (Vibrant Life, 1999). People who suffered from osteoarthritis, rheumatoid arthritis, yfibromylagia, joint injuries, and osteoporosis have been treated successfully with SAM-e (Glorioso et al., 1985; Marcolongo et al., 1985; DiPadova, 1987; König, 1987; Maccagno et al., 1987; Vetter, 1987). A dozen European clinical trials involving more than 22,000 patients have found SAM-e to be effective for treatment of joint pain and inflammation from arthritis.Side effects include occasional gastrointestinal disturbances, mainly diarrhea6. in methylation reactions that aid in the production of cartilage proteoglycans.13A number of studies have found SAMe to be more effective than placebo in improving pain and stiffness related to osteoarthritis. ${ }^{15-19}$ No studies documenting disease arrest or reversal are found in the literature $^{20}$. But it is found that it can treat for some extent.

\section{CONCLUSION}

SAMe though it is a super nutrient it should be taken with an prescription only as most of the effects it caused is unknown till now, and many more studies are need to be done on this, especially its studies should be conducted on liver regeneration effect of it, and has it shows promising effects on treating depression studies should be done to make it as a perfect drug.

\section{ACKNOWLEDGEMENT:}

It is here to inform that I shivashankarpursuing m.pharmacy(dept. of pharmaceutics) in Anurag Pharmacy Collage, that all the review work as done by myself alone, and I may be responsible for any disputes arise. 


\section{REFERENCE}

1. Bottiglieri T. S-Adenosyl-L-methionine (SAMe): from the bench to the bedside--molecular basis of a pleiotrophic molecule. Am J ClinNutr2002; 76: 1151S-1157S 8 36. ConsumerLab.com. Product review: SAMe. [http://www.consumerlab. com]. Accessed March 11, 2002.

2. The review of natural products. Drugs Facts and Comparisons. St. Louis, Mo.: Facts and Comparisons, 1996.

3. summaryrepor t Prepared by Technical Resources International, Inc. under contract no. NO2-CB-50511 (11/99)

4. Lu SC, Mato JM. Role of methionine adenosyltransferase and SAMe in alcohol-associated liver cancer. Alcohol 2005;35:227234.

5. Cantoni, G. L (1975) Annu. Rev. Biochem. U, 435-451

6. Tabor, C. W. and Tabor, H. (1984) Adv. Enzymol. 56, 251-282

7. Baldessarini RJ. Neuropharmacology of S-adenosyl-Lmethionine. Am J Med 1987;83:95-103.

8. Stramentinoli G, et al. Intestinal absorption of S-adenosyl-Lmethionine. J PharmacolExpTher1979;209:323-326.

9. Bell KM, et al. S-adenosylmethionine blood levels in major depression: Changes with drug treatment. ActaNeurolScandSuppl1994;154:15-18.

10. Bottiglieri T, et al. Cerebrospinal fluid S-adenosylmethionine in depression anddementia: Effects of treatment with parenteral and oral S-adenosylmethionine. JNeurolNeurosurg Psychiatry 1990;53:1096-1098.

11. Gaster B. S-adenosylmethionine (SAMe) for treatment of depression. Altern Med Alert 1999; 2:133-5.
12. Hepatoprotective effects of $\mathrm{S}$-adenosyl-L-methionine against alcohol- and cytochrome P450 2E1-induced liver injury; World J Gastroenterol2010 March 21; 16(11): 1366-1376 ;ISSN 1007 9327 (print)

13. Presenilin 1 gene silencing by $\mathrm{S}$-adenosylmethionine: a treatment for Alzheimer disease? FEBS 27136 FEBS Letters 541 (2003) $145^{\wedge} 148$

14. Safety and efficacy of S-adenosylmethionine (SAMe) for osteoarthritis

15. Di Padova C. S-adenosylmethionine in the treatment of osteoarthritis. Review of the clinical studies. Am J Med 1987;83:60-5

16. Bradley JD, Flusser D, Katz BP, Schumacher HR Jr, Brandt KD, Chambers MA, et al. A randomized, double blind, placebo controlled trial of intravenous loading with Sadenosylmethionine (SAM) followed by oral SAM therapy in patients with knee osteoarthritis. J Rheumatol 1994;21:905-11.

17. Barcelo HA, Wiemeyer JC, Sagasta CL, Macias M, Barreira JC. Effect of $\mathrm{S}$ adenosylmethionine on experimental osteoarthritis in rabbits. Am J Med 1987;83:55-9.

18. Konig B. A long-term (two years) clinical trial with Sadenosylmethionine for the treatment of osteoarthritis. Am J Med 1987;83:89-94.

19. Srivastava KC, Mustafa T. Ginger (Zingiberofficinale) in rheumatism and musculoskeletal disorders. Med Hypotheses 1992;39:342-8.

20. The review of natural products. Drugs Facts and Comparisons. St. Louis, Mo.: Facts and Comparisons, 1996

21. 